Case Report

\title{
Legionnaires' Disease Complicated with Rhabdomyolysis and Acute Kidney Injury in an AIDS Patient
}

\author{
Karan Seegobin, Satish Maharaj, Cherisse Baldeo, Julio Perez Downes, and Pramod Reddy \\ Department of Internal Medicine, University of Florida College of Medicine, Jacksonville, FL 32209, USA \\ Correspondence should be addressed to Karan Seegobin; karanseegobin@hotmail.com
}

Received 8 May 2017; Revised 22 August 2017; Accepted 5 September 2017; Published 4 October 2017

Academic Editor: Alexandre R. Marra

Copyright (C) 2017 Karan Seegobin et al. This is an open access article distributed under the Creative Commons Attribution License, which permits unrestricted use, distribution, and reproduction in any medium, provided the original work is properly cited.

\begin{abstract}
Objective. To present a case of an uncommon triad of Legionella pneumonia, rhabdomyolysis, and renal failure, with review of the relevant literature. Case. A 51-year-old with a history of human immunodeficiency virus (HIV), chronic obstructive pulmonary disease (COPD), and hypertension presented with fever, cough, and shortness of breath over four days. Chest X-ray showed consolidation in left lower lung field; urine was positive for Legionella antigen and myoglobin; creatine kinase was 51092U/L; creatine was $6.9 \mathrm{mg} / \mathrm{dL}$, and his CD4 count was 41 cells/ul. He was managed with azithromycin and levofloxacin and further required dialysis and ventilatory support in the intensive care unit due to renal failure and respiratory failure. He responded well to the treatment and made a complete recovery. Legionella pneumophila infection is a recognized but rare cause of rhabdomyolysis with high morbidity and mortality when there is extrapulmonary involvement. Early diagnosis and appropriate treatment is essential to improve outcomes. Conclusion. Physicians should consider Legionella pneumonia in patients with rhabdomyolysis, renal failure, and respiratory symptoms. Early diagnosis and treatment have been shown to have good clinical response. Timely intensive care management, together with early and judicious use of dialysis in patients complicated with rhabdomyolysis and renal failure, may lead to good outcomes.
\end{abstract}

\section{Introduction}

Legionella pneumophila (LP) infection is a recognized but rare cause of rhabdomyolysis $[1,2]$. It can be further complicated with renal impairment which can be due to acute tubular necrosis (ATN) or acute tubulointerstitial nephritis (ATIN) [3]. This triad of pneumonia, renal failure, and rhabdomyolysis is associated with high morbidity and mortality [4]. We report a case of an immunocompromised patient with Legionella pneumonia complicated with acute kidney injury and rhabdomyolysis. He required dual antibiotics with levofloxacin and azithromycin, in addition to dialysis, and intensive care treatment. He made a full recovery with normal renal function. After review of the relevant literature on reported cases of this uncommon triad of Legionella pneumonia, renal failure, and rhabdomyolysis, early diagnosis has been shown to have good clinical response. We advocate for timely transfer to the intensive care unit (ICU) and judicious use of dialysis in patients with complicated Legionella pneumophilia as the outcomes are good.

\section{Case}

A 51-year-old male with a past medical history of HIV, COPD, and hypertension presented with a four-day history of fever, shortness of breath, and nonproductive cough associated with headache and reduced appetite. He had a 30pack-year history of smoking cigarettes and had not been compliant with his HIV medications as well as trimethoprimsulfamethoxazole. He had no history of nonsteroidal antiinflammatory drug (NSAID), herbal drug, or cocaine use. $\mathrm{He}$ denied recent ill contacts, recent travel, or camping. On examination, he was in respiratory distress with blood pressure $143 / 95 \mathrm{mmHg}$, pulse 135 beats per minute, respiratory rate 24 breaths per minute, temperature 39.5 degrees Celsius, and sPO2 $92 \%$ on room air and $98 \%$ on 2 litres nasal cannula. $\mathrm{He}$ had bronchial breath sounds in the left mid and lower lung fields with crackles, but no wheezing. His heart sounds were normal. Abdomen was soft and nontender, with normal bowel sounds. Other aspects of his examination were unremarkable. 


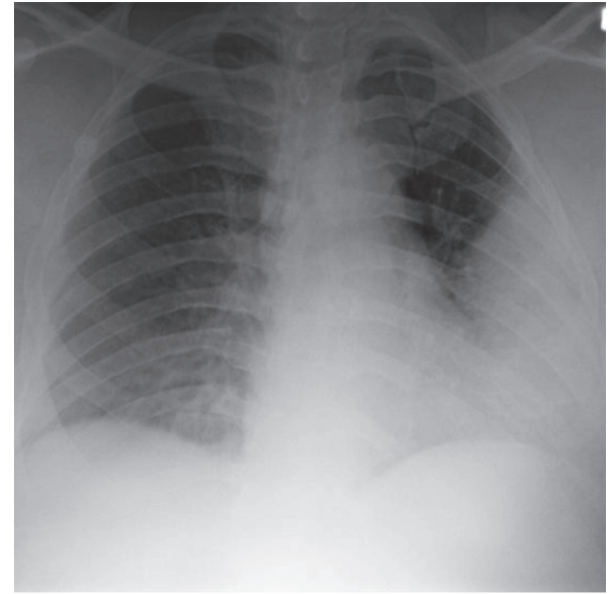

FIGURE 1: Chest X-ray with homogenous consolidation in the left lower lung field.

His white cell count was $4.6\left(4.5-11 \times 10^{3} / \mathrm{uL}\right)$; haemoglobin was 10.7 (12-16 g/dL); platelet was 246 (140-440 thou/ cu mm); mean corpuscular volume was 94 (82-101 fl); Prothrombin Time (PT) was 12.9 (11-13.5 seconds); international normalised ratio (INR) was 1.0 (0.8-1.2); partial thromboplastin time (PTT) was 34 (25-35 seconds); HbAlc was $5.7 \%$ (4-5.6\%); procalcitonin was $31.2 \mathrm{ng} / \mathrm{mL}$ $(<0.15 \mathrm{ng} / \mathrm{mL})$; thyroid stimulating hormone $(\mathrm{TSH})$ was $0.705(0.27-4.2 \mathrm{uIU} \cdot \mathrm{ml})$; sodium was $137(135-145 \mathrm{mmol} / \mathrm{L})$; potassium was $4.5(3.3-4.6 \mathrm{mmol} / \mathrm{L})$; chloride was $95(101-$ $110 \mathrm{mmol} / \mathrm{L}) ; \mathrm{BUN}$ was $56(6-22 \mathrm{mg} / \mathrm{dL})$; creatine was 6.98 $(0.6-1.17 \mathrm{mg} / \mathrm{dL})$; calcium was $7.8(8.6-10 \mathrm{mg} / \mathrm{dL})$; albumin was $2.6(3.8-4.9 \mathrm{~g} / \mathrm{dL})$; phosphorus was $5.5(2.5-4.5 \mathrm{mg} / \mathrm{dL})$; aspartate aminotransferase was 789 (14-33 IU/L); ALT was 235 (10-42 IU/L); anion gap was 22 (4-16); direct bilirubin was $3.3(0-0.2 \mathrm{mg} / \mathrm{dL})$; indirect bilirubin was $0.4 \mathrm{mg} / \mathrm{dL}$ $(0.2-1.2 \mathrm{mg} / \mathrm{dL})$; total bilirubin was $3.7(0.2-1.0 \mathrm{mg} / \mathrm{dL})$; alkaline phosphatase was 66 (40-129 IU/L); total creatine kinase was $51092 \mathrm{U} / \mathrm{L}(22-195 \mathrm{U} / \mathrm{L})$, and urine was positive for myoglobin. His CD 4 count was 41 cells/ul, and HIV viral load was 34900 copies $/ \mathrm{mL}$.

Urine analysis with Alere BinaxNOW lateral flow immunochromatographic assay for Legionella antigen serogroup 1 was positive; urine pneumococcal antigen was negative; blood culture, respiratory culture, and urine culture were without bacterial growth; respiratory viral panel was reported negative for influenza, parainfluenza, rhinovirus, respiratory syncytial virus (RSV) virus, human metapneumovirus, $\mathrm{H} 1$, and $\mathrm{H} 3$.

His electrocardiogram showed sinus tachycardia. Chest $\mathrm{X}$-ray (CXR) showed a homogenous consolidation in the left lower lung field (Figure 1). CT chest showed consolidation in the left lingual with air bronchograms consistent with lobar pneumonia, without pleural effusion (Figures 2 and 3). Echocardiograph showed a left ventricular ejection fraction of $65 \%$. Renal ultrasound showed normal sized kidneys with normal echogenicity without hydronephrosis.

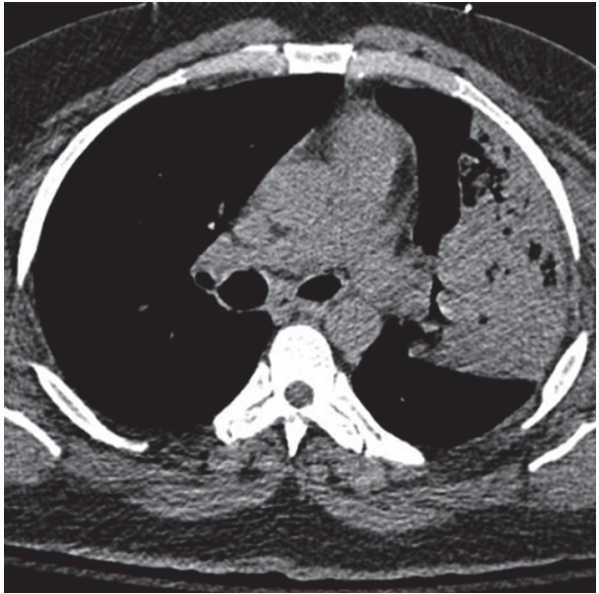

FIGURE 2: Axial section chest CT showing consolidation in the left lingual with air bronchograms consistent with lobar pneumonia, without pleural effusion.

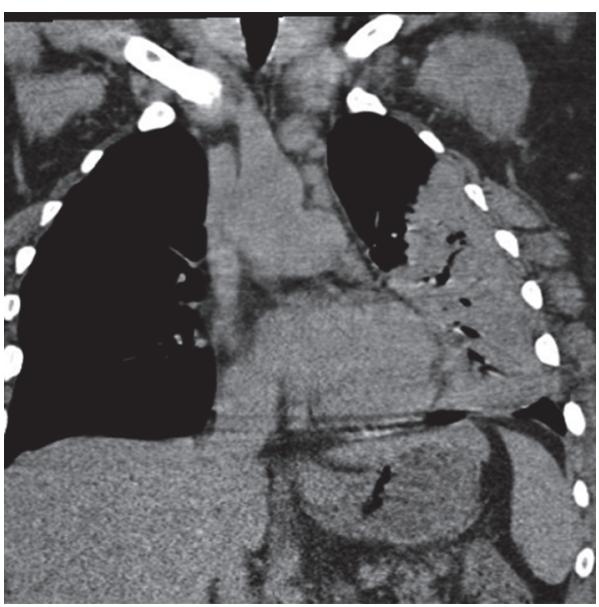

FIgURE 3: Coronal section chest CT showing consolidation in the left midzone with air bronchograms consistent with lobar pneumonia, without pleural effusion.

He was diagnosed with Legionella pneumonia, rhabdomyolysis, and acute renal failure and started on levofloxacin and atovaquone. He became further oliguric, with rising creatine and BUN, with worsening respiratory status requiring intubation and ventilation in addition to dialysis which were commenced on the second day of admission. Azithromycin was added to his antibiotic regimen in the ICU. His maximum creatine was $13.04 \mathrm{mg} / \mathrm{dL}$, and BUN was $153 \mathrm{mg} / \mathrm{dL}$ during his hospital course. His respiratory and renal function improved during his ICU stay. He was extubated after six days. With improvement in his urine output and renal indices; he no longer required dialysis. On discharge, after 28 days of hospital stay and seven days of ICU stay, his creatine was $2.6 \mathrm{md} / \mathrm{dL}$, BUN $36 \mathrm{md} / \mathrm{dL}$, total creatine kinase (CK) $69 \mathrm{U} / \mathrm{L}$, aspartate aminotransferase (AST) $16 \mathrm{IU} / \mathrm{L}$, alanine aminotransferase (ALT) $30 \mathrm{IU} / \mathrm{L}$, direct bilirubin $0.1 \mathrm{mg} / \mathrm{dL}$, indirect bilirubin $0.1 \mathrm{mg} / \mathrm{dL}$, and 
TABLE 1

\begin{tabular}{|c|c|c|c|c|c|}
\hline Reference & Age (years) & $\begin{array}{l}\text { Dialysis } \\
\text { required }\end{array}$ & $\begin{array}{l}\text { ICU treatment } \\
\text { needed }\end{array}$ & $\begin{array}{c}\text { Antimicrobial class (quinolone, } \\
\text { macrolide, or both quinolone } \\
\text { and macrolide) }\end{array}$ & Outcome \\
\hline Koufakis et al. [5] & 45 & Yes & Yes & Quinolone and macrolide & Recovery \\
\hline Shimura et al. [3] & 54 & Yes & Yes & Quinolone and macrolide & Recovery \\
\hline McConkey et al. [1] & 56 & No & Yes & Quinolone & Recovery \\
\hline Shah et al. [6] & 26 & Yes & Undetermined & Macrolide & Recovery \\
\hline Erdogan et al. [4] & 67 & Yes & Undetermined & Quinolone and macrolide & Recovery \\
\hline Abe et al. [7] & 56 & Yes & Yes & Quinolone and macrolide & Died \\
\hline $\begin{array}{l}\text { Wiegele and Krenn } \\
\text { [8] }\end{array}$ & 44 & Yes & Yes & Undetermined & Recovery \\
\hline Linga and Deo [2] & 40 & Undetermined & Yes & Undetermined & Recovery \\
\hline Agu et al. [9] & 45 & No & Undetermined & Quinolone & Recovery \\
\hline Nakatani et al. [10] & 50 & Yes & Undetermined & Quinolone & Recovery \\
\hline Li et al. [11] & 55 & Yes & Undetermined & Quinolone and macrolide & Recovery \\
\hline Daumas et al. [12] & 55 & Yes & Undetermined & Quinolone and macrolide & Recovery \\
\hline Narita et al. [13] & 48 & Yes & Yes & Quinolone & Recovery \\
\hline Sposato et al. [14] & 61 & Yes & Yes & Macrolide & Died \\
\hline Matsumoto et al. [15] & 67 & Yes & Yes & Macrolide & Recovery \\
\hline Tokuda et al. [16] & 57 & Undetermined & Yes & Macrolide & Died \\
\hline
\end{tabular}

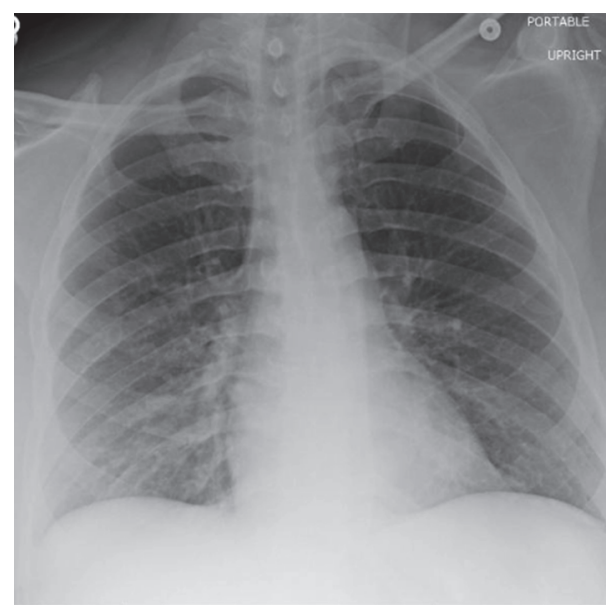

FIGURE 4: CXR after three-week follow-up, without consolidation.

total bilirubin $0.2 \mathrm{mg} / \mathrm{dL}$. After three-weeks follow-up in the outpatient setting, his creatine was back to normal at 1.06 $(0.6-1.17 \mathrm{mg} / \mathrm{dL})$, with resolution of the chest X-ray consolidation (Figure 4). Renal biopsy was not pursued in light of recovery of normal renal function.

\section{Discussion}

In adults, Legionella causes $2-15 \%$ of community acquired pneumonia (CAP) cases that require hospitalization [5]. It is the second most common cause of serious pneumonia that needs admission in an intensive care unit (ICU) [5]. The first report that associated Legionella and rhabdomyolysis was published in 1980 by Posner et al. [5] It is a recognized but rare cause of rhabdomyolysis [1, 2]; a clinical syndrome characterized by elevated serum concentrations of creatine phosphokinase $(\mathrm{CPK})$ and myoglobinuria leading to renal dysfunction [17].

Renal impairment in Legionella pneumophila infection accompanied by rhabdomyolysis can be due to ATN or ATIN [3]. Legionellosis-associated ATIN could be either indirectly associated with L pneumophila via rhabdomyolysis or directly affected by L pneumophila [3]. Interestingly, renal anomalies can develop days before the imaging demonstration of pneumonia [18].

Many of the laboratory findings are nonspecific and include renal and hepatic dysfunction, hyponatremia, hypophosphatemia, thrombocytopenia, leukocytosis, hematuria, and proteinuria [1]. The gold standard is culture and sensitivity on specialized charcoal media, which has a sensitivity of $70-80 \%$ [1]. However, this is not a rapid test and usually takes 3-5 days [1]. Urine antigen test is a rapid, practical, and inexpensive method for the diagnosis of the disease, characterized by sensitivities of $70-90 \%$ and specificities approaching $100 \%[4,5]$. In our case the use of urine antigen testing confirmed the etiology of the pneumonia.

From a search on PubMed and Google with the terms "Legionella", "rhabdomyolysis", and "renal failure" we analysed case reports (Table 1 ) of 16 patients with the triad of renal failure, rhabdomyolysis, and Legionella pneumonia with focus on CK levels, need for dialysis, intensive care admission, outcomes after treatment, and the use of quinolones, macrolides, or both.

Thirteen patients made full recovery, five of which were treated with a quinolone and macrolide, with the others having either a quinolone or macrolide as part of their treatment. Observational studies suggest that quinolones are 
more likely to achieve a favourable outcome in terms of patient survival and length of hospitalization [5]. Combined treatment is believed to be superior to monotherapy in cases of severe clinical disease or in immunosuppressed subjects [5]. In our case early treatment with a quinolone and macrolide led to good outcome.

Of the 16 patients analysed, 8 patients had CK levels greater than $5,000 \mathrm{U} / \mathrm{L}$ at presentation, with four out of those 8 having levels $>20,000 \mathrm{U} / \mathrm{L}$. Our patient also had a significantly high CK level at $51092 \mathrm{U} / \mathrm{L}$. Considering that patients present to the hospital days after the onset of disease, this may give the bacteria sufficient time to cause muscle injury. The exact mechanism of muscle injury caused by Legionella is still unclear [19]. However, release of an endotoxin or exotoxin that causes rhabdomyolysis and direct bacterial invasion seem to be the most probable mechanisms [19]. In another report published in 1992 that studied renal failure in patients with Legionella disease, 8 patients had rhabdomyolysis out of 45 cases, three of which died; 2 of which did not undergo dialysis [6]. Physicians should always anticipate rhabdomyolysis and check for its presence in patients with Legionella disease, as early anticipation of this event and initiation of early aggressive fluid resuscitation are critical in reducing the risk of myoglobinuric acute kidney injury [20]. CK levels have been shown to correlate well with severity of rhabdomyolysis in some reports [21]. Furthermore, in one study of 1,769 adult patients, serum CK > $773 \mathrm{U} / \mathrm{L}$ correlated well with the onset of acute kidney injury (AKI) and rhabdomyolysis [21]. Many of the patients from our analysis in addition to our patient had the presence of renal impairment with CK levels > $773 \mathrm{U} / \mathrm{L}$ [22].

From our analysis of 16 patients, most cases required ICU care (10 cases). A 1998 study of 392 cases of community acquired pneumonia revealed $12.5 \%$ due to Legionella and $20.4 \%$ of those requiring ICU admission [1]. It is reported that chronic lung disease, immunodeficiency, malignancies, renal impairment, diabetes mellitus, nosocomial acquisition, and delayed initiation of specific antimicrobial therapy are associated with poor outcomes [5]. Our patient had four of these factors which could have predisposed him to a more severe course of Legionella disease. Additionally, it is reported that late but not early admission to the ICU for community acquired pneumonia is associated with poorer outcomes in comparison to patients managed on medical wards [23]. From our analysis, of the 10 patients admitted to the ICU, seven had good outcomes. Our patient also had a good recovery. It is important that there be early identification of patients who may develop severe Legionella pneumonia within the very first days of hospital stay to intensify the level of care and to propose early ICU transfer as the outcomes may be better.

From this review of 16 patients, most of these patients became progressively oliguric with worsening renal indices during their hospital course eventually requiring dialysis as seen in 12 of these cases. Our patient took a similar course despite aggressive fluid resuscitation. With this trend, frequent monitoring of renal indices and urine output is critical in patients with Legionella disease in light of the propensity for this disease to follow the path of eventually requiring dialysis, as seen in our case. The timing of initiating dialysis is a challenge and should not be determined solely by myoglobin or CK serum concentration but by the status of renal impairment, with complications such as lifethreatening hyperkalemia, hypercalcemia, hyperazotemia, anuria, or hyperhydration without response [21].

It is generally accepted that patients with immune paresis, especially cell-mediated, are susceptible to Legionella infection [24]. Nevertheless, Legionella pneumophila is not commonly described in persons with infection due to human immunodeficiency virus (HIV) [25]. There is no clear relation between incidence of Legionella CAP and CD4 count [26, 27]. In a 10-year retrospective single center study, 15 cases of Legionella CAP were observed. When compared with pneumococcal CAP, disease was more severe with Legionella with a higher incidence of bilateral pneumonia, respiratory failure, and need for ventilatory support. Extra respiratory symptoms, hyponatremia, and increased creatine phosphokinase were more frequent in Legionella pneumonia [27]. Our patient was not compliant with prophylactic trimethoprimsulfamethoxazole (TMP-SMX). This drug has some activity against Legionella and in previous reports there is a low incidence of Legionella pneumonia in persons on TMPSMX prophylaxis [25]. Health professionals should have high suspicion for Legionella as a possible etiology for pneumonia, in AIDS patients with TMP-SMX noncompliance.

\section{Conclusion}

Consider Legionella pneumonia in patients presenting with rhabdomyolysis, renal failure, and respiratory symptoms. Early diagnosis and appropriate treatment have been shown to have good clinical response. Timely intensive care management, together with early and judicious use of dialysis, in patients complicated with rhabdomyolysis and renal failure may lead to good outcomes.

\section{Disclosure}

Karan Seegobin assumes responsibility for the integrity of the contents.

\section{Conflicts of Interest}

The authors have no potential areas of conflict to declare.

\section{Authors' Contributions}

The idea for reporting this case was that of Karan Seegobin. Further intellectual content and editing were done by all authors. All authors saw, edited, and approved the final contents.

\section{References}

[1] J. McConkey, M. Obeius, J. Valentini, and M. S. Beeson, "Legionella pneumonia presenting with rhabdomyolysis and acute renal failure: A case report," Journal of Emergency Medicine, vol. 30, no. 4, pp. 389-392, 2006. 
[2] K. R. Linga and D. B. Deo, "Pneumonia, rhabdomyolysis and acute renal Failure - a Deadly Cocktail!", American Journal of Respiratory and Critical Care Medicine, vol. 189, A1778 pages, 2014.

[3] C. Shimura, T. Saraya, H. Wada et al., "Pathological evidence of rhabdomyolysis-induced acute tubulointerstitial nephritis accompanying Legionella pneumophila pneumonia," Journal of Clinical Pathology, vol. 61, no. 9, pp. 1062-1063, 2008.

[4] H. Erdogan, A. Yilmaz, O. Kal, A. Erdogan, and H. Arslan, "Rhabdomyolysis-induced acute renal failure associated with legionnaires' disease," Scandinavian Journal of Urology and Nephrology, vol. 40, no. 4, pp. 345-346, 2006.

[5] T. Koufakis, I. Gabranis, M. Chatzopoulou, A. Margaritis, and M. Tsiakalou, "Severe Legionnaires' disease complicated by rhabdomyolysis and clinically resistant to moxifloxacin in a splenectomised patient: too much of a coincidence?" Case Reports in Infectious Diseases, vol. 2015, pp. 1-4, 2015.

[6] A. Shah, F. Check, S. Baskin, T. Reyman, and R. Menard, "Legionnaires' disease and acute renal failure: case report and review," Clinical Infectious Diseases, vol. 14, no. 1, pp. 204-207, 1992.

[7] M. Abe, K. Kaizu, and K. Matsumoto, "Clinical evaluation of pneumonia-associated rhabdomyolysis with acute renal failure," Therapeutic Apheresis and Dialysis, vol. 12, no. 2, pp. 171175, 2008.

[8] M. Wiegele and C. G. Krenn, "Cytosorb in a patient with legionella-pneumonia associated rhabdomyolysis," ASAIO Journal, vol. 61, no. 3, pp. e14-e16, 2015.

[9] C. Agu, M. Basunia, and D. Salhan, "Legionella pneumonia associated with severe rhabdomyolysis and acute kidney injury," American Journal of Respiratory and Critical Care Medicine, vol. 193, A7164 pages, 2016.

[10] S. Nakatani, H. Inariba, Y. Kumeda et al., "A case of Legionella pneumonia associated with acute renal failure," Nihon Toseki Igakkai Zasshi, vol. 43, no. 4, pp. 381-386, 2010.

[11] J. Li, R. Wen, H. Deng, and Q. Li, "A case of Legionella pneumonia with multiple organ failure," Journal of Central South University (Medical Sciences), vol. 41, no. 6, pp. 657-660, 2016.

[12] A. Daumas, F. El-Mekaoui, S. Bataille et al., "Acute tubulointerstitial nephritis complicating Legionnaires' disease: a case report," Journal of Medical Case Reports, vol. 6, article 100, 2012.

[13] Y. Narita, K. Naoki, N. Horiuchi et al., "A case of legionella pneumonia associated with acute respiratory distress syndrome (ARDS) and acute renal failure treated with methylprednisolone and sivelestat," Nihon Kokyūki Gakkai Zasshi: The Journal of the Japanese Respiratory Society, vol. 45, no. 5, pp. 413-418, 2007.

[14] B. Sposato, S. Mariotta, A. Ricci, G. Lucantoni, and G. Schmid, "Legionnaire's pneumonia with rhabdomyolysis and acute renal failure. a case report," Recenti Progressi in Medicina, vol. 94, no. 9, pp. 391-394, 2003.

[15] N. Matsumoto, H. Mukae, S. Yamashita et al., "A case of severe Legionnaires' disease complicated by rhabdomyolysis, acute renal failure, liver dysfunction and encephalopathy," Kansenshogaku zasshi. The Journal of the Japanese Association for Infectious Diseases, vol. 74, no. 11, pp. 989-993, 2000.

[16] H. Tokuda, N. Yahagi, S. Kasai, S. Kitamura, and Y. Otsuka, "A case of fatal pneumonia caused by Legionella pneumophila serogroup 6 developed after drowning in a public bath," Kansenshogaku zasshi. The Journal of the Japanese Association for Infectious Diseases, vol. 71, no. 2, pp. 169-174, 1997.
[17] G. H. Malik, "Rhabdomyolysis and myoglobin-induced acute renal failure," Saudi Journal of Kidney Diseases and Transplantation, vol. 9, pp. 273-284, 1999.

[18] U. Singh and W. M. Scheld, "Infectious etiologies of rhabdomyolysis: three case reports and review," Clinical Infectious Diseases, vol. 22, no. 4, pp. 642-649, 1996.

[19] J. Clement, P. Maes, and M. Van Ranst, "Acute kidney injury in emerging, non-tropical infections," Acta Clinica Belgica, vol. 62, no. 6, pp. 387-395, 2007.

[20] O. S. Better and Z. A. Abassi, "Early fluid resuscitation in patients with rhabdomyolysis," Nature Reviews Nephrology, vol. 7, no. 7, pp. 416-422, 2011.

[21] N. Petejova and A. Martinek, "Acute kidney injury due to rhabdomyolysis and renal replacement therapy: a critical review," Critical Care, vol. 18, no. 3, article 224, 2014.

[22] M. El-Ebiary, X. Sarmiento, A. Torres, and etal., "Prognostic factors of severe Legionella pneumonia requiring admission to ICU," American Journal of Respiratory and Critical Care Medicine, vol. 156, pp. 1467-1472, 1997.

[23] B. Renaud, C. Brun-Buisson, A. Santin et al., "Outcomes of early, late, and no admission to the intensive care unit for patients hospitalized with community-acquired pneumonia," Academic Emergency Medicine, vol. 19, no. 3, pp. 294-303, 2012.

[24] D. Schlossberg and J. Bonoan, "Legionella and immunosuppression," Seminars in Respiratory Infections, vol. 13, no. 2, pp. 128$131,1998$.

[25] S. P. Blatt, M. J. Dolan, C. W. Hendrix, and G. P. Melcher, "Legionnairesș disease in human immunodeficiency virusinfected patients: eight cases and review," Clinical Infectious Diseases, vol. 18, no. 2, pp. 227-232, 1994.

[26] U. Sandkovsky, G. Sandkovsky, J. Suh, B. Smith, V. Sharp, and B. Polsky, "Legionella pneumonia and HIV: case reports and review of the literature," AIDS Patient Care and STDs, vol. 22, no. 6, pp. 473-481, 2008.

[27] L. M. Pedro-Botet, N. Sopena, A. García-Cruz et al., "Streptococcus pneumoniae and Legionella pneumophila pneumonia in HIV-infected patients," Scandinavian Journal of Infectious Diseases, vol. 39, no. 2, pp. 122-128, 2007. 


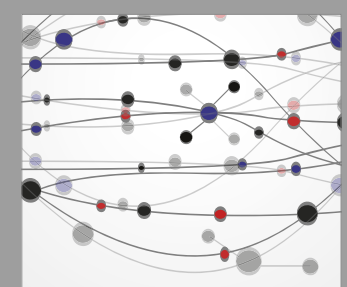

The Scientific World Journal
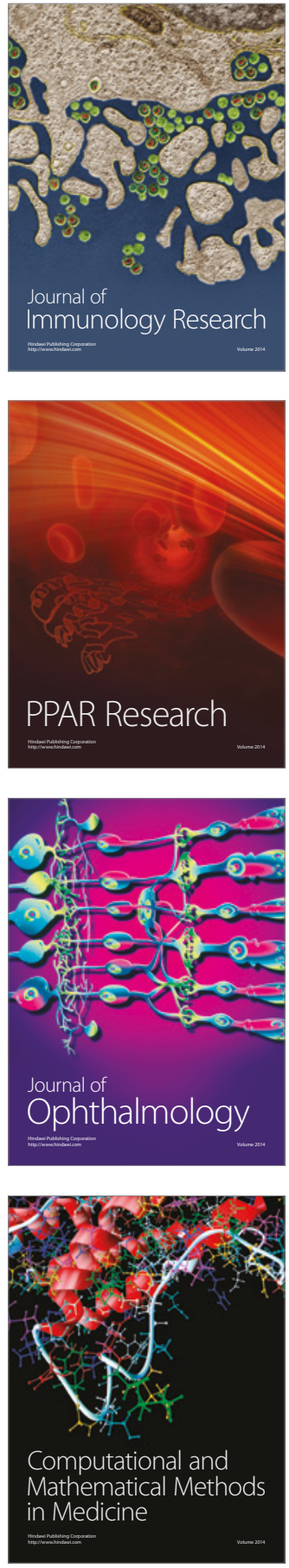

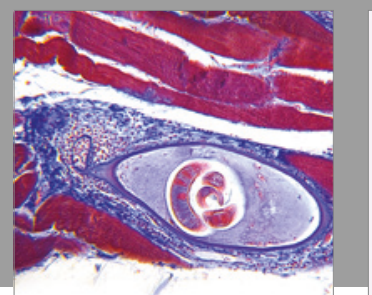

Gastroenterology Research and Practice
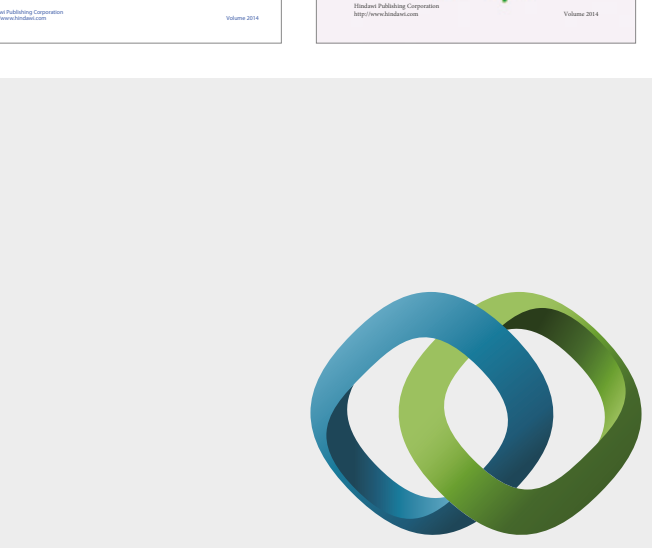

\section{Hindawi}

Submit your manuscripts at

https://www.hindawi.com
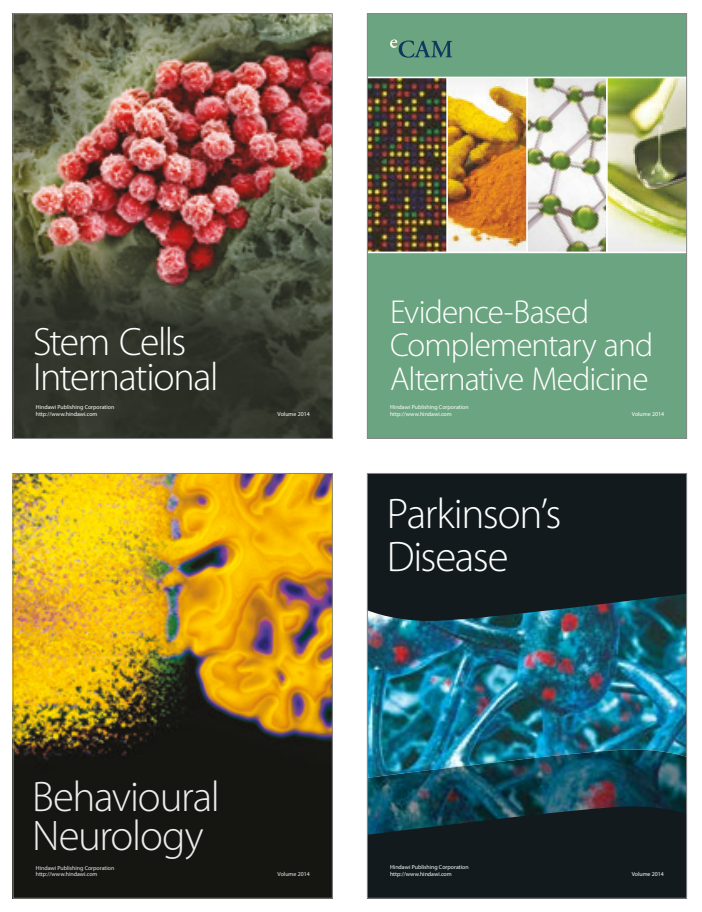
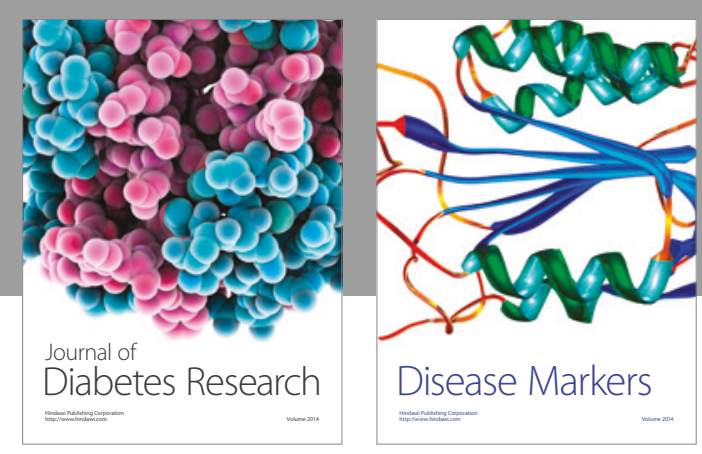

Disease Markers
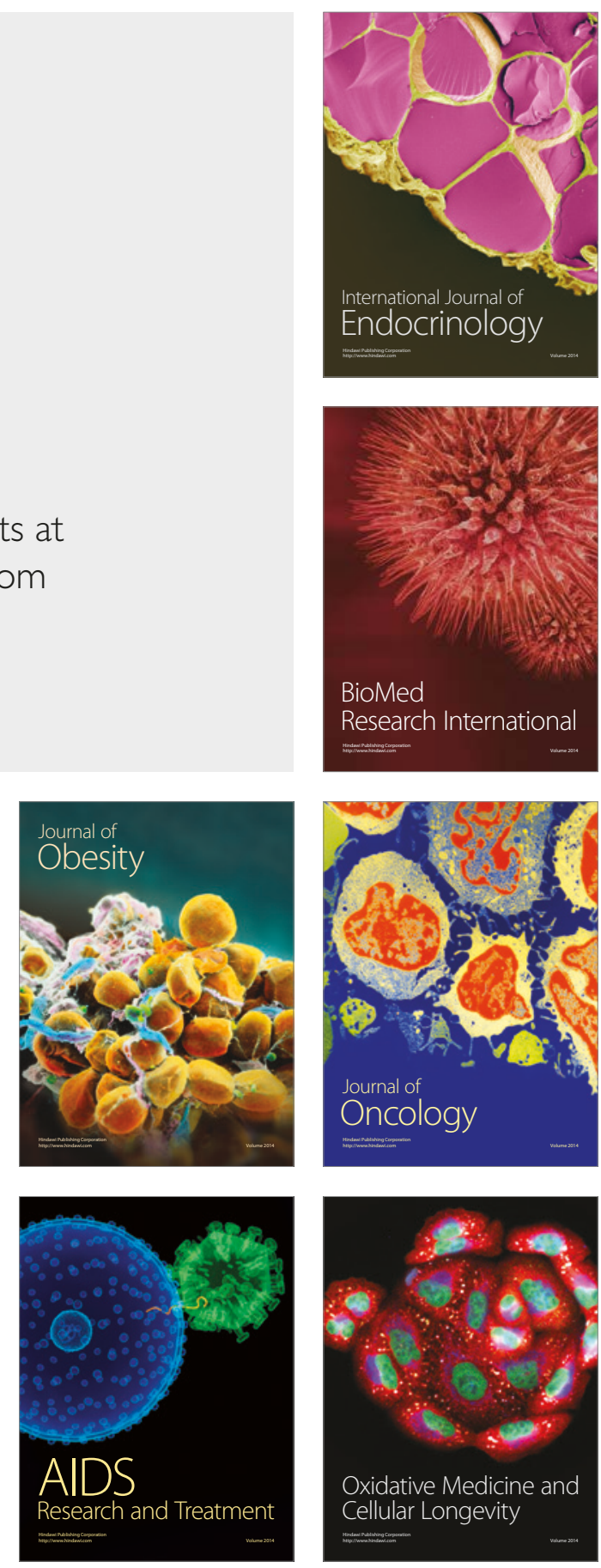